\title{
The mTOR Inhibitor Everolimus Attenuates the Time Course of Chronic Anti-Thy1 Nephritis in the Rat
}

\author{
Sandra Wittmann ${ }^{a}$ Christoph Daniel ${ }^{\mathrm{a}}$ Andrea Braun $^{\mathrm{a}}$ Regina Vogelbacher ${ }^{\mathrm{a}}$ \\ Fuijo Shimizu $^{\text {b Hiroshi Kawachi }}{ }^{\text {b Christian Hugo }}{ }^{a}$ \\ ${ }^{a}$ Department of Nephrology and Hypertension, University of Erlangen-Nuremberg, Erlangen, Germany; \\ ${ }^{b}$ Department of Cell Biology, Institute of Nephrology, Niigata University Graduate School of Medical and \\ Dental Sciences, Niigata, Japan
}

\section{Key Words}

Chronic kidney disease - Everolimus - Matrix deposition • mTOR inhibitor $\cdot$ Vascular endothelial growth factor

\begin{abstract}
Background: The antiproliferative immunosuppressant everolimus adversely affects the acute reversible anti-Thy 1 nephritis model. We hypothesized that everolimus treatment started after the acute proliferative phase could even be beneficial in the chronic anti-Thy 1 nephritis model in the rat. Methods: Chronic anti-Thy 1 nephritis was induced by injection of the monoclonal antibody $1-22-3$ in 20 male Sprague-Dawley rats 7 days after uninephrectomy. Two weeks after disease induction, rats were randomly treated with either everolimus or vehicle for 14 weeks. Changes in progression of renal disease were investigated by immunohistochemistry and real-time PCR 16 weeks after disease induction. Results: During chronic anti-Thy 1 nephritis, the formation of focal segmental glomerulosclerosis lesions, the degree of interstitial fibrosis as well as the increase in proteinuria over 14 weeks was ameliorated by everolimus treatment. Increased glomerular hypertrophy observed in the vehicle-treated rats was completely prevented in the everolimus-treated nephritic rats. Increased glomerular fibronectin mRNA and protein as well as the renal influx of monocytes/macrophages was significantly reduced in the evero-
\end{abstract}

\section{KARGER}

Fax +41 613061234

E-Mail karger@karger.ch

www.karger.com (c) 2008 S. Karger AG, Basel

$1660-2129 / 08 / 1082-0045 \$ 24.50 / 0$

Accessible online at:

www.karger.com/nee limus group. Everolimus reduced the pro-angiogenic factor vascular endothelial growth factor (VEGF) and VEGF mRNA in glomeruli, while the transforming growth factor- $\beta$ signaling pathway was not affected. Conclusion: 'Late' start of everolimus treatment demonstrates beneficial effects on the time course of chronic anti-Thy 1 nephritis.

Copyright @ 2008 S. Karger AG, Basel

\section{Introduction}

During the last years, the mammalian target of rapamycin (mTOR) inhibitors, such as everolimus and sirolimus, were frequently used as potent immunosuppressants in renal and cardiac transplant therapy due to the lack of nephrotoxicity as well as potential antitumor effects [1-3]. Nevertheless, while several clinical renal transplant studies have suggested the beneficial effects of calcineurin inhibitor-free, mTOR inhibitor-based protocols, unexpected adverse effects of mTOR inhibitors have also been described during treatment of renal allograft nephropathy [4-6], human glomerulonephritis [7], as well as experimental kidney disease $[8,9]$. The mTOR inhibitors are potent antiproliferative agents for lymphocytes, and also for other cell types such as vascular smooth muscle cells [1], endothelial cells [10] and mesangial cells [11]. Everolimus is a lipophilic macrocyclic lactone bind-
Sandra Wittmann, University Erlangen-Nuremberg, Medical Clinic IV

Department of Nephrology and Hypertension

Loschgestrasse 8, DE-91054 Erlangen (Germany)

Tel. +49 9131853 9206, Fax +499131 8539202

E-Mail sandra.wittmann@med4.imed.uni-erlangen.de 
ing to the FK-binding protein 12 , while this complex then inhibits mTOR and thereby arrests the cell cycle in the transition of G1 to $S$ phase. These antiproliferative effects are considered to be especially beneficial for hyperproliferative lesions such as cardiac transplant vasculopathy, vascular restenosis after balloon dilatation or, theoretically, in mesangial proliferative glomerulonephritides such as IgA nephropathy. In contrast, we recently demonstrated the detrimental effects of everolimus therapy in an experimental rat model of acute mesangial proliferative glomerulonephritis, the anti-Thyl model, in which everolimus treatment unexpectedly transformed this acute reversible model into a chronic disease model with sclerosis in more than $30 \%$ of the glomeruli [8]. While this acute reversible anti-Thyl model perfectly demonstrates the successful healing reaction of severely injured glomeruli with involvement of both the glomerular endothelium and the mesangium, the situation of human mesangial proliferative glomerulonephritis may be better matched by the chronic anti-Thyl model. This model is induced after uninephrectomy with antibody 122-3 [12-14], and has a very similar time course during the first 2 weeks to the acute anti-Thyl model. Here, early mesangial injury with severe mesangiolysis and glomerular endothelial injury, with the frequent formation of glomerular microaneurysms, are followed by a coordinated repair reaction by mesangial and endothelial migration and proliferation. The glomerular architecture is substantially restored 2 weeks after disease induction and characterized by hypercellular lesions with increased matrix expansion. While in the acute anti-Thyl model glomeruli return to an almost completely normal structure during the next few months, the time course of the chronic anti-Thyl model after the first 2 weeks is characterized by progressive proteinuria, matrix accumulation, tubulointerstitial lesions and focal segmental glomerulosclerosis (FSGS)-like lesions similar to human FSGS. Considering the evidence from the acute anti-Thyl model [8], where early treatment was most detrimental, we examined whether a late start of everolimus therapy at the end of the acute proliferative phase may even lead to beneficial effects in the chronic anti-Thyl model.

\section{Methods}

Animal Model and Experimental Design

The animal studies were performed in accordance with the internal animal review board (Regierung von Mittelfranken: 6212531.31-17/05). Male Sprague-Dawley rats (Charles River, Sulzfeld, Germany) weighing 105-145 g were used for this study. The

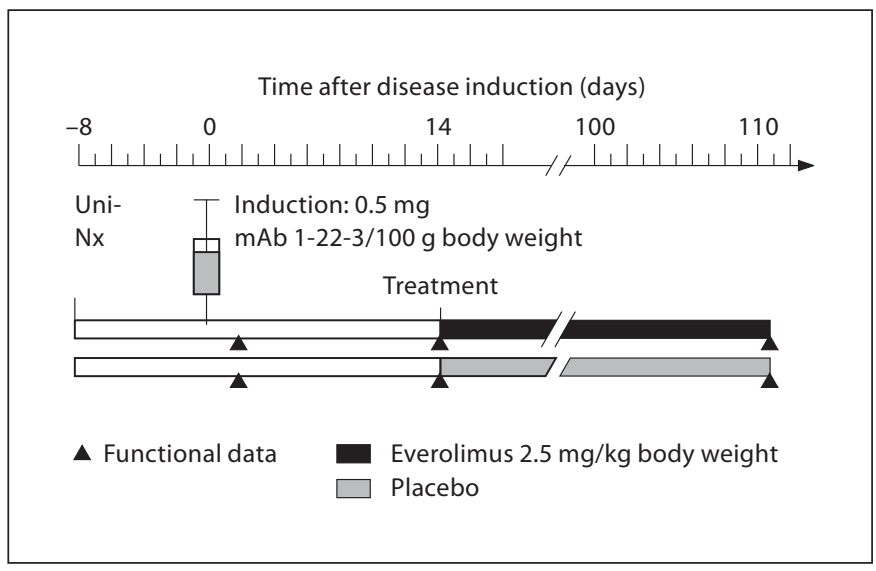

Fig. 1. Experimental design of the in vivo study.

animals were fed standard rat chow (Altromin 1324, Spezialfutterwerke $\mathrm{GmbH}$, Lage, Germany) and tap water ad libitum.

Seven days after uninephrectomy, experimental chronic mesangial proliferative glomerulonephritis (chronic anti-Thyl model) was induced by an injection of the monoclonal anti-Thyl antibody 1-22-3 (0.5 mg/100 g body weight) [12-14] (fig. 1). To evaluate the best starting point for everolimus therapy, 3 rats each were investigated in a pilot experiment before and on days 7 and 14 after induction of chronic anti-Thyl nephritis. At the beginning of treatment on day 14 the animals were randomized after measuring proteinuria into 2 groups. Microemulsion formulations (Self-micro-emulsifying-drug-delivery system) of vehicle and everolimus for oral administration were provided by Novartis Pharmaceuticals, Inc. (Basel, Switzerland) and were diluted in phosphate-buffered saline. Vehicle or everolimus were given once daily orally via gavage at a dose of $2.5 \mathrm{mg} / \mathrm{kg}$ body weight. This dose resulted in trough levels of $7.6 \pm 2.25 \mathrm{ng} / \mathrm{ml}$ as determined by tandem mass spectrometry, which was consistent with other studies [15]. Since the adverse effects of everolimus treatment have been described during the acute phase of the anti-Thyl model, everolimus treatment was started 14 days after induction of the chronic anti-Thyl disease model. At that chosen time point, the typical mesangial and endothelial lesions as indicated by mesangiolysis and microaneurysm formation were almost completely repaired via cellular proliferation and migration (fig. 2). All rats were randomly allocated to two different groups with chronic mesangial proliferative nephritis $(\mathrm{n}=10)$, one vehicle- and one everolimus-treated (fig. 1). A serum sample and 24-hour urine collection for measuring proteinuria and serum creatinine were done at periodic intervals. After 8 weeks the systolic blood pressure was recorded using the tail-cuff plethysmographic method. The rats were sacrificed after 17 weeks (119 days) and the tissue samples were processed and stored as needed.

Renal Morphology and Immunohistochemistry

Tissue for light microscopy was fixed in methyl Carnoy's solution, embedded in paraffin, and cut into $2-\mu \mathrm{m}$ sections for periodic acid Schiff reagent (PAS) or indirect immunoperoxidase staining as described elsewhere [16, 17]. 

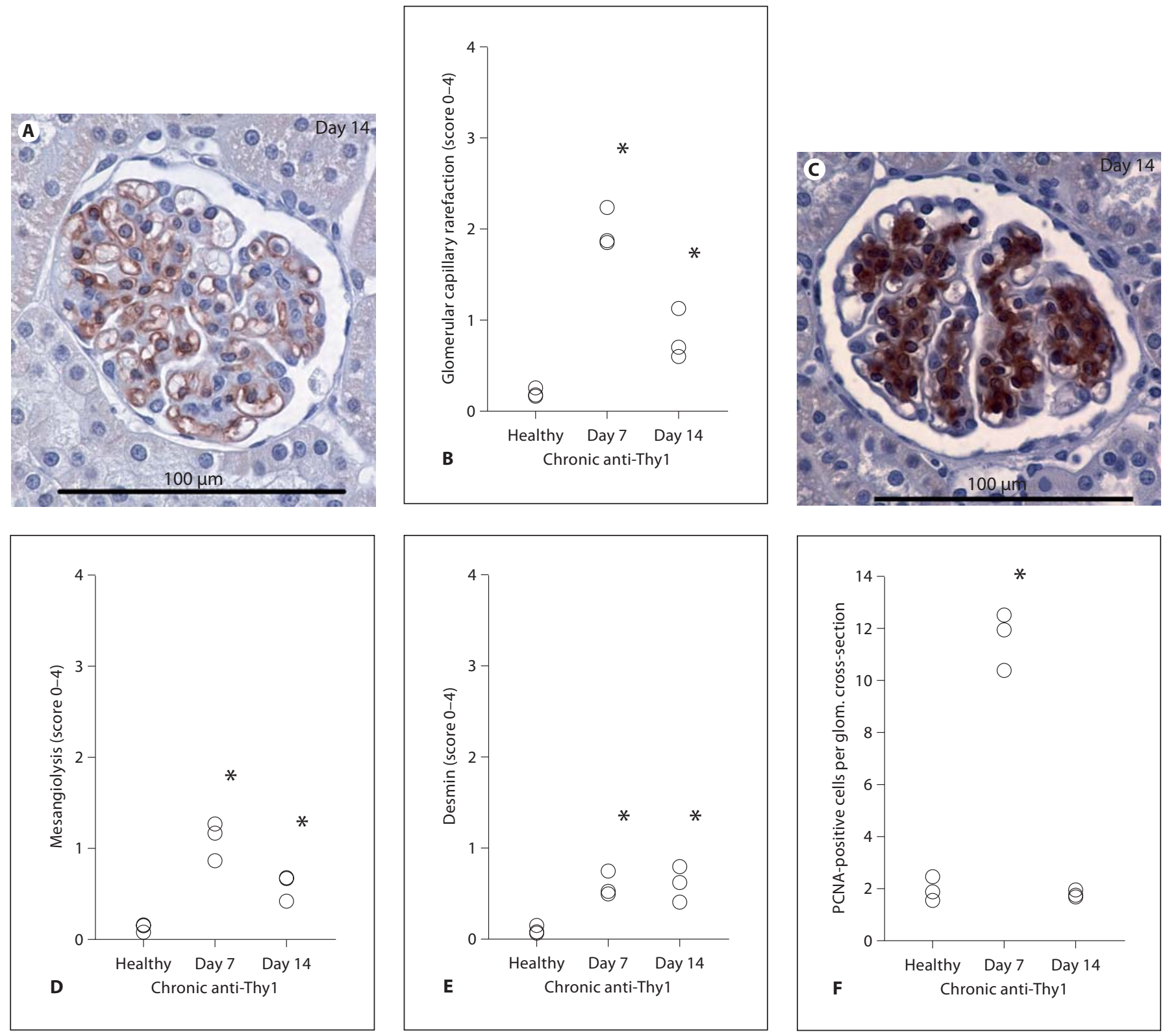

Fig. 2. Characteristics of chronic anti-Thyl nephritis in the rat on day 14 at the starting point of therapy. A Representative glomerular endothelial cell staining (dark grey color, JG12 stain) on day 14. B, D Glomerular capillary rarefaction (semiquantitative scoring system, B) as well as mesangial injury (semiquantitative mesangiolysis score, D) were almost completely reconstituted on day 14. C Mesangial restoration and hypercellularity on day 14 of the chronic anti-Thyl model. E Podocyte injury, as shown by desmin positivity of the outer glomerular capillary rim (immunohistochemical staining), was increased during disease but similar on days 7 and 14. F Glomerular proliferation, as assessed by the number of PCNA-positive cells per glomerular cross-section, almost completely ceased on day 14 .
A FSGS lesion was defined by a scarred segmental injury of the glomerulus that includes synechia between the tuft and Bowman's capsule. The interstitial fibroses score $(0-4)$ was defined as the grade of tubule dilatations, PAS positivity and inflammatory cells $(0=$ normal, $1=$ tubular dilatations or inflammatory cells, $2=$ tubular dilatations and PAS positivity $<25 \%, 3=$ tubular dila- tations and PAS positivity $>25 \%$, and $4=$ tubular dilatations and PAS positivity $>50 \%$ ).

The following antibodies were used in this study: PC10, a murine IgG1 monoclonal antibody (mAb) against the human proliferating cell nuclear antigen (PCNA), cross-reactive with rat (DAKO, Glostrup, Denmark) [16, 17]; ED-1, a murine monoclonal 
IgG1 $\mathrm{mAb}$ to a rat cytoplasmic antigen present in monocytes, macrophages, and dendritic cells (Linaris, Wertheim-Bettingen, Germany) [16, 17]; sc-152, a rabbit polyclonal antibody to human vascular endothelial growth factor (VEGF)-A cross-reactive with rat VEGF (Santa Cruz Biotechnology, Santa Cruz, Calif., USA) [18]; JG12, a murine IgG mAb specific for rat aminopeptidase P on endothelial cells (Bender MedSystems GmbH, Vienna, Austria) $[19,20]$; sc-8418, a murine monoclonal antibody against rat p70S6 kinase (Santa Cruz Biotechnology) [21]; sc-8416, a murine monoclonal antibody to phosphorylated p70S6 kinase recommended for detection in rats (Santa Cruz Biotechnology) [21]; a rabbit polyclonal antibody against rat fibronectin (FN; Chemicon, Temecula, Calif., USA) [22]; a murine monoclonal antibody for human desmin (DAKO, Glostrup, Denmark); a murine IgG $\mathrm{mAb}$ against the Thyl epitope specific for rat mesangial cells (clone Ox-7; Serotec Ltd, Oxford, UK) [23], and monocyte chemotactic protein-1 (MCP-1), a goat polyclonal antibody against a peptide mapping at the C-terminus of MCP-1 of the rat (sc-1785; Santa Cruz Biotechnology).

Negative controls for immunostaining included either deleting the primary antibody or substitution of the primary antibody with equivalent concentrations of an irrelevant murine $\mathrm{mAb}$ or pre-immune rabbit IgG. For each biopsy, 30-60 glomerular cross-sections (400- to 600 -fold magnification) or 20 visual fields (200-fold magnification) were evaluated in a blinded fashion. Glomerular expression of VEGF was graded semiquantitatively and reflected changes in the area and intensity of mesangial staining. Glomerular expression of FN, VEGF and MCP-1 was quantified by a semiquantitative scoring system $(0=0 \%, 1=<25 \%, 2=<50 \%, 3=<75 \%, 4=>75 \%)$. In the case of immunostaining for desmin, only the podocytes on the outer edge of the glomerular tuft were assessed as described elsewhere [24]. A semiquantitative staining score from 0 to 4 was used to represent the percentage ranges of the outer edge showing positive staining $(0=0 \%, 1=1-25 \%, 2=26-50 \%, 3=51-75 \%, 4=$ 76-100\%). The lack of immunostaining of either the endothelium (JG12) or the mesangium (Ox-7) was evaluated by a semiquantitative scoring system from 0 to $4(0=$ normal capillary/ mesangial structure, $1=<25 \%, 2=<50 \%, 3=<75 \%, 4=>75 \%$ loss of endothelium/mesangium) reflecting glomerular capillary rarefaction or mesangiolysis. The glomerular cell number was counted in the immunostaining of phosphorylated p70S6 kinase. Glomerular hypertrophy was evaluated by computerized measurement of the glomerular area using the MetaVue Imaging System (Visitron Systems GmbH, Puchheim, Germany). It has been shown previously that this semiquantitative scoring system is reproducible between different observers and that the data obtained correlate with those obtained by computerized morphometry $[18,25]$.

\section{Isolation of Glomeruli}

This method was performed in a cold storage room $\left(4^{\circ} \mathrm{C}\right)$. After withdrawal of the kidney the renal cortex was minced with a razor blade, pressed using a pusher through a sieve with a mesh size of $106 \mu \mathrm{m}^{2}$ and rinsed with phosphate-buffered saline. Larger contaminations were retained on a second sieve with a mesh size of $180 \mu \mathrm{m}^{2}$ and glomeruli were collected on a third sieve with a mesh size of $75 \mu \mathrm{m}^{2}$. Small contaminations washed through all sieves. The collected glomeruli were transferred to a sterile blue cap and concentrated by centrifugation at $1,000 \mathrm{rpm}$ for $5 \mathrm{~min}$.
The glomeruli were lysed in RLT buffer for RNA isolation (Qiagen RNeasy Mini Kit).

Quantification of Glomerular TGF- $\beta 1, V E G F, P A I-1$ and FN by Real-Time PCR

RNA isolation (Qiagen RNeasy Mini Kit) and reverse transcription (Abgene, UK) were performed according to the manufacturer's instructions. The cDNA of transforming growth factor$\beta 1$ (TGF- $\beta 1$ ), VEGF, plasminogen activator inhibitor-1 (PAI-1) and FN was amplified and quantified by real-time PCR (ABI Prism 7000, Applied Biosystems), using the Taqman/SYBR (Abgene, UK) assay reagent methods and the comparative threshold cycle method. TGF- $\beta 1$, VEGF, PAI- 1 and FN mRNA expression was normalized to $\beta$-actin mRNA. For this PCR, $3 \mu$ l of each cDNA sample was mixed with $17 \mu \mathrm{l}$ Taqman/SYBR Master Mix, which includes the $2 \times$ Taqman/SYBR solution, target primers, RNAse-free water, and the equal probe for the Taqman method. The final concentration for the forward and reverse primers in the Taqman reaction was $900 \mathrm{nM}$ and for the probe $250 \mathrm{nM}$. By contrast, in the SYBR the reaction was used at a final concentration for both primers of $100 \mathrm{nM}$. The results were reproducible.

The following rat-specific PCR-primers were used in this study.

\section{$\beta$-Actin [26] \\ Forward: 5' CGCGAGAAGATGACCCAGATCATG 3' \\ Reverse: 5' AGGATCTTCATGAGGTAGTCAGT 3'}

PAI-1 [26]

Forward: 5' TTTGGGAAAGGGTTCCCTTC 3'

Reverse: 5' AGTGGTTGATGATGAATCTGG 3'

TGF- $\beta 1[27]$

Forward: 5' TGGAAGTGGATCCACGCGCCCAAGG 3'

Reverse: 5' GCAGGAGCGCACGATCATGTTGGAC 3'

VEGF [28]

Forward: 5' AACGAAAGCGCAAGAAATCC 3'

Reverse: 5' GCTCACAGTGAACGCTCCAG 3'

\section{FN}

Forward: 5' ACATCAGTGAATGCCAGTCCTTT 3'

Reverse: 5' TCAGAACCGGAACGGAGAAA 3'

Probe: $5^{\prime}$ TGGTTCAGACTGCAGTGACCAACATTGA 3'

\section{Miscellaneous Measurements}

Urinary protein was measured using the BioRad Protein Assay (BioRad, Munich, Germany) and BSA (Pierce, Bonn, Germany) as standard in concentrations from 10 to $500 \mu \mathrm{g} / \mathrm{ml}$ according to the manufacture's instructions.

\section{Statistical Analysis}

The Kolmogorov-Smirnov test was used to test normality. The result showed that there was no normal distribution in all the parameters analyzed. So the statistical significance was evaluated using the Mann-Whitney test. The outcomes are displayed as box plots or dot plots. The box plots and dot plots were drawn in SPSS. A box plot is a convenient way of graphically depicting the 5-number summary. The box represents $50 \%$ of all events, whereby the line in the box shows the median. The 'whiskers' (vertical bars) 

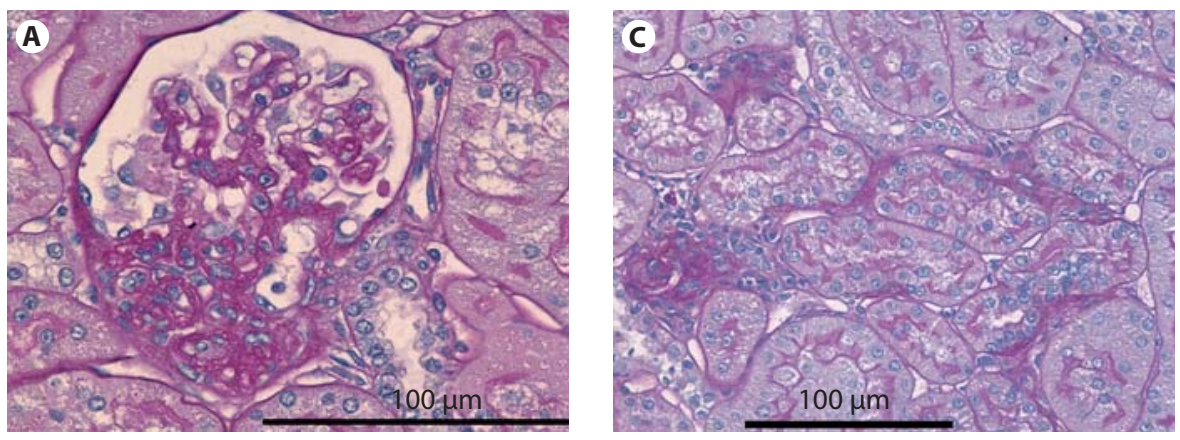

Fig. 3. Everolimus prevents glomerular FSGS lesions and tubulointerstitial fibrosis in chronic anti-Thyl nephritis. A Typical picture of a FSGS lesion (PAS staining). B The percentage of FSGS lesions 16 weeks after nephritis induction was significantly reduced by everolimus therapy. ${ }^{*}$ Significant changes at $p<0.05$. C A representative example for interstitial fibrosis is shown by PAS staining. D Interstitial fibrosis was assessed by semiquantitative scoring of PASstained tissue sections and showed an almost significant reduction in everolimus treated nephritic rats $(\mathrm{p}=0.05)$.
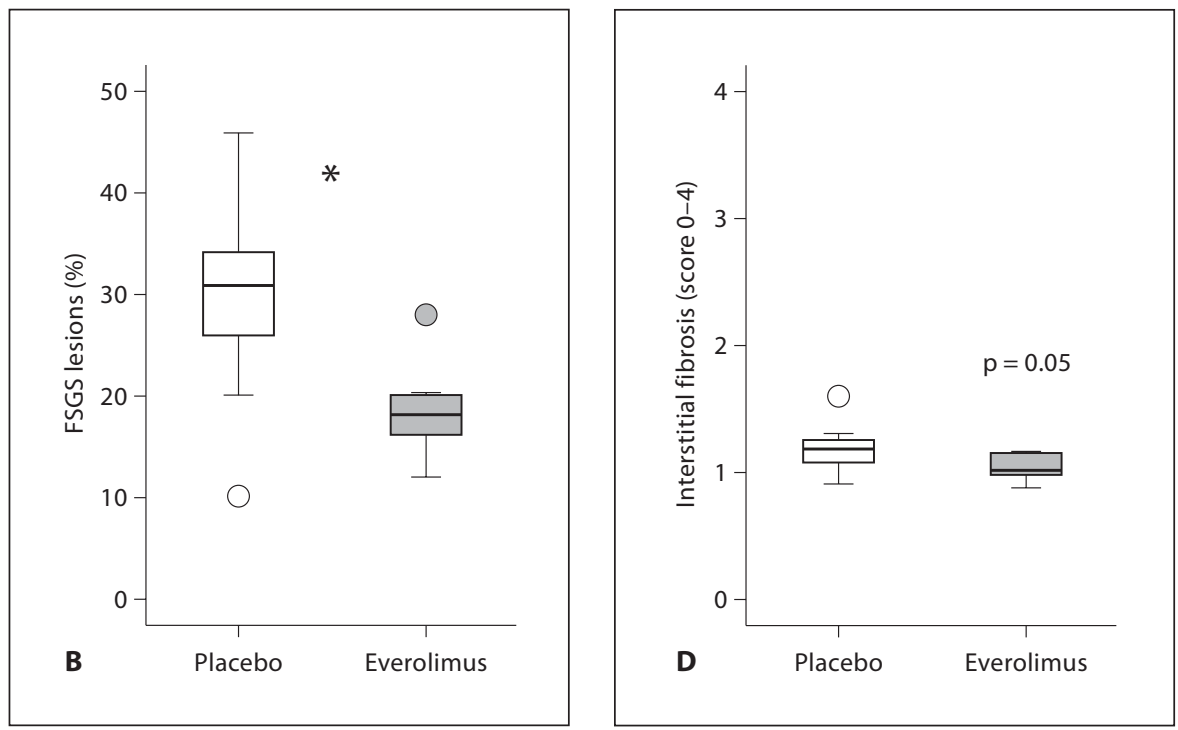

extend to at most 1.5 times the box length. They must end at an observed value, thus connecting all the values outside the box that are not more than 1.5 times the box length away from the box. Three times the box length marks the boundary between 'mild' and 'extreme' outliers (shown as dots). All values in this text are expressed as mean $\pm \mathrm{SD}$.

\section{Results}

\section{Everolimus Treatment Was Started at a Time when} the Glomerular Architecture Was Almost Restored

Since adverse effects of everolimus treatment have been described during the acute phase of the anti-Thyl model, everolimus treatment was started 14 days after disease induction of the chronic anti-Thyl model. At that chosen time point, the typical mesangial and endothelial lesions, as indicated by mesangiolysis and microaneurysm formation, were almost completely repaired via cellular proliferation and migration, as shown in a represen-

Everolimus in a Chronic Anti-Thyl Model tative aminopeptidase $\mathrm{P}$ staining on endothelial cells (JG12; fig. 2A) and Ox-7 staining for the mesangium (fig. 2C). Semiquantitative scoring of glomerular capillary rarefaction from 0 to 4 revealed quite prominent endothelial damage on day 7 after model induction, reaching values of $2.0 \pm 0.22$. Fourteen days after nephritis induction, the appearance of microaneurysms was very rare and glomerular capillary rarefaction declined to a score of $0.8 \pm 0.28$, indicating nearly complete reconstitution of the endothelium (fig. 2B). In parallel, mesangiolysis was reduced from a score $1.1 \pm 0.21$ on day 7 to nearly complete mesangial restoration on day 14 (score $0.6 \pm 0.14$ ), as measured by Ox-7 positivity of the glomeruli (fig. 2D). At earlier time points (days 1-2), mesangiolysis scores up to 3.5-4 can be observed [8]. Indirect damage of podocytes, as quantified by immunostaining against desmin, was relatively low and not different on days 7 and 14 after induction of chronic anti-Thyl nephritis (fig. 2E). On day 7, glomerular proliferative activ- 


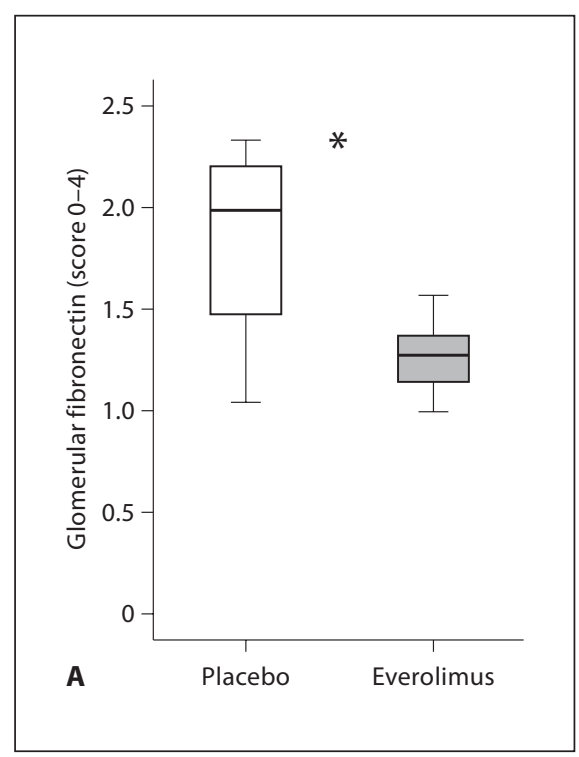

Fig. 4. Everolimus inhibits production and deposition of glomerular fibronectin without affecting TGF- $\beta$ signal transduction. A Glomerular fibronectin was analyzed by semiquantitative scoring of immunohistologic staining in placebo- and everolimustreated rats. ${ }^{*}$ Significant changes at $\mathrm{p}<$ 0.05 . B Fibronectin mRNA was reduced by everolimus as evaluated in isolated glomeruli using real-time PCR. * Significant changes at $\mathrm{p}<0.05$. C, E Glomerular TGF- $\beta$ mRNA (C) and mRNA for the TGF- $\beta$ target gene plasminogen activator inhibitor-1 (PAI-1; E) were analyzed in isolated glomeruli by real-time PCR and were not different in both groups. D The glomerular percentage of $\mathrm{P}$-smad 2/3-positive cells, a signal transduction molecule of TGF- $\beta$, was also unaffected by everolimus therapy as assessed by immunostaining.
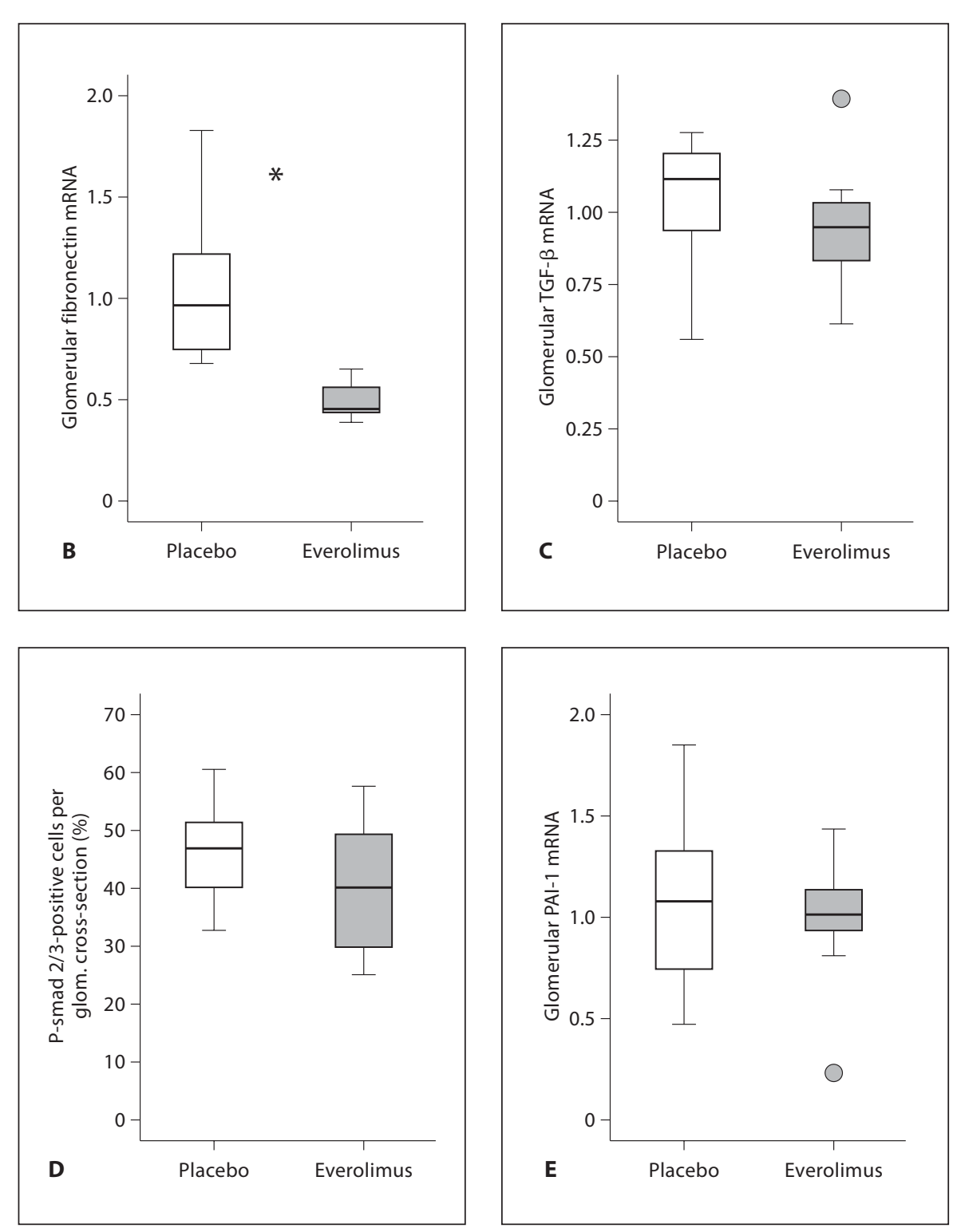

ity was six times higher than in healthy rats, but returned to normal values on day 14 after disease induction, as shown by staining for PCNA, indicating that repair by proliferative activity was completed (fig. $2 \mathrm{~F}$ ).

\section{Everolimus Prevents FSGS Lesions in Chronic \\ Anti-Thy1 Nephritis}

A typical end point in chronic mesangioproliferative nephritis is the formation of FSGS lesions as defined by scarred segmental injury of the glomerulus that includes synechia between the tuft and Bowman's capsule (fig. 3A). Sixteen weeks after model induction, everolimus treat- ment reduced the formation of FSGS lesions by 35\% compared to animals treated with vehicle (fig. 3B). Damage of podocytes, as analyzed by immunostaining against desmin, was increased twofold (not significant, $p=0.2$ ) in vehicle-treated animals (score $1.2 \pm 0.8$ ) compared to the evaluation on days 7 (score $0.6 \pm 0.1$ ) and 14 (score 0.6 \pm 0.1 ; fig. $2 \mathrm{E}$ ). In contrast, everolimus treatment resulted in podocyte damage (score $0.68 \pm 0.68$ ) equivalent to early time points of chronic anti-Thyl nephritis on days 7 and 14 (fig. 2E). Tubulointerstitial fibrosis was relatively low, as shown in figure 3C, and was almost significantly reduced $(\mathrm{p}=0.05)$ by everolimus therapy (fig. 3D). 

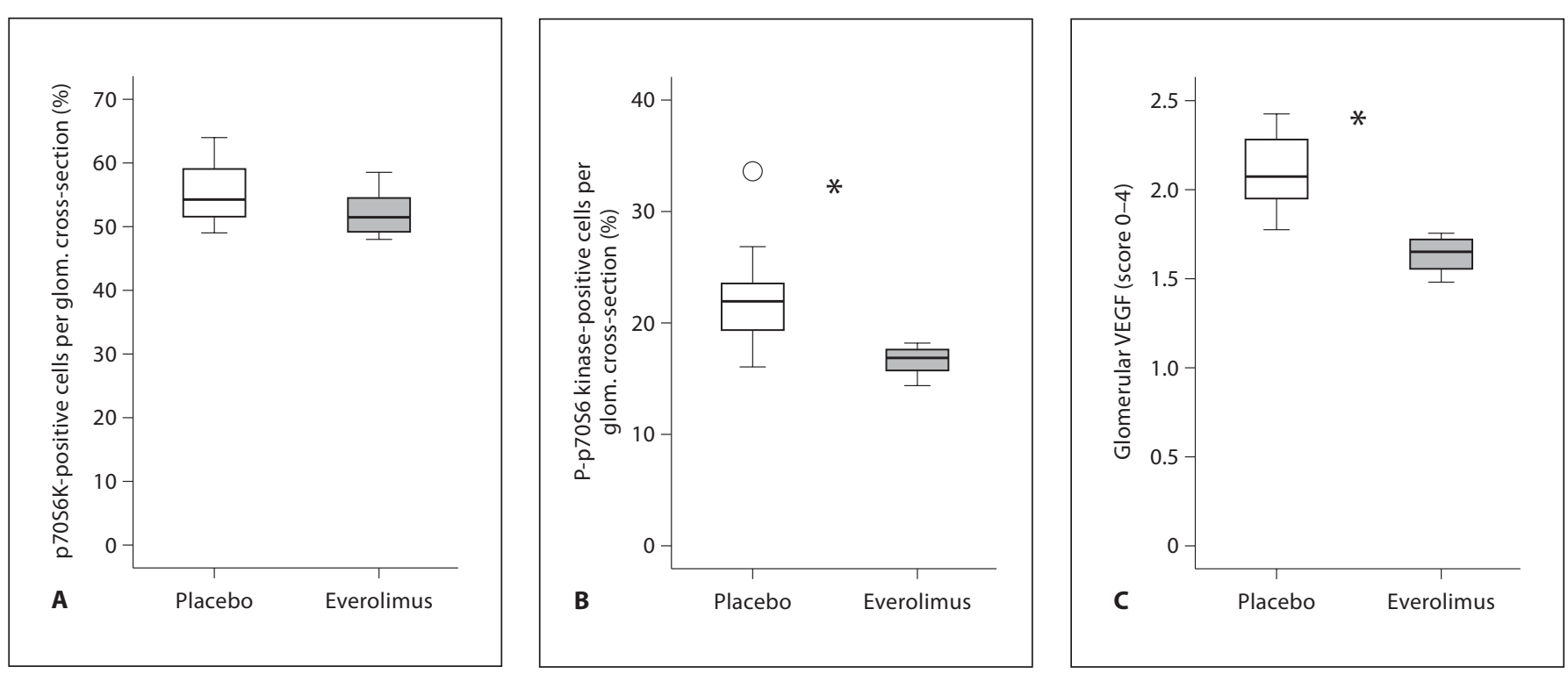

Fig. 5. Everolimus inhibits VEGF production and deposition and phosphorylation of p70S6 kinase. A, B The percentage of nuclei positive for glomerular p70S6 kinase (A) or phosphorylated p70S6 kinase (B) was evaluated by immunostaining indicating a selectively inhibited phosphorylation of glomerular p70S6 kinase by everolimus. ${ }^{*}$ Significant changes at $\mathrm{p}<0.05$. C, D Everolimus significantly reduced both glomerular VEGF protein, as assessed by immunostaining (C) and VEGF mRNA expression as quantified by real-time PCR (D). ${ }^{*}$ Significant changes at $\mathrm{p}<0.05$. E The glomerular endothelium was analyzed by a capillary rarefaction score using JG12 immunostaining and was not affected long-term.
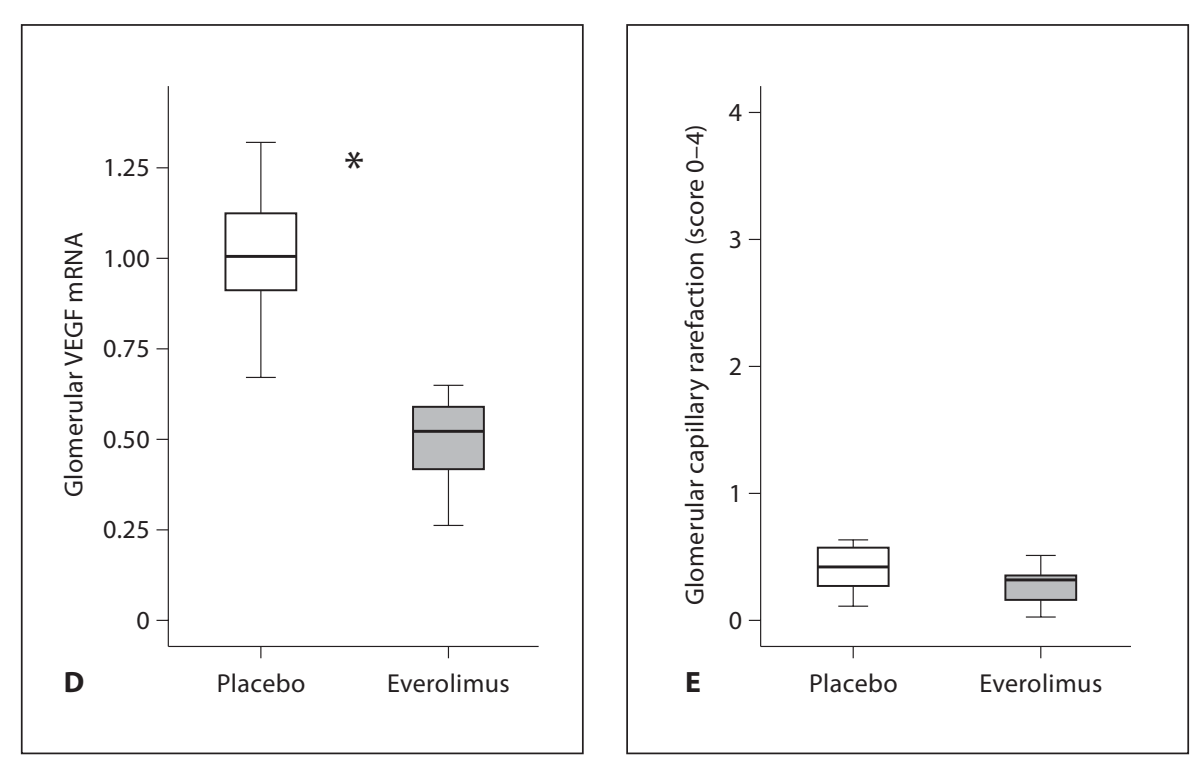

Everolimus Reduces Glomerular Deposition and

Expression of FN in Chronic Anti-Thy1 Nephritis

Glomerular FN, a matrix protein, was significantly decreased by $30 \pm 9 \%$ in the everolimus-treated group as shown by the semiquantitative scoring system (fig. 4A). In addition, glomerular FN mRNA was significantly downregulated by $50 \pm 8 \%$ in the everolimus-treated group (fig. 4B). The most important pro-fibrotic cytokine in renal disease is TGF- $\beta$. To investigate the potential influence of everolimus on the TGF- $\beta$ signal transduction system we analyzed TGF- $\beta 1$ mRNA expression, the TGF- $\beta$ signal transduction molecule Smad $2 / 3$ and the TGF- $\beta$

Everolimus in a Chronic Anti-Thyl Model downstream target PAI-1. Neither expression of TGF- $\beta 1$ itself (fig. $4 \mathrm{C}$ ), nor phosphorylation of the TGF- $\beta$ signal transduction molecule Smad 2/3 (fig. 4D), nor expression of PAI-1 (fig. 4E) was affected by everolimus therapy.

Everolimus Inhibits VEGF and Phosphorylation of the p70S6 Kinase during Chronic Anti-Thy1 Nephritis

Everolimus binds to FK-binding protein 12 and this complex inhibits mTOR, which affects the phosphorylation of p70S6 kinase. Expression of glomerular p70S6 kinase, as analyzed by immunostaining, was not influenced by everolimus (fig. 5A). In contrast, phosphorylation of 

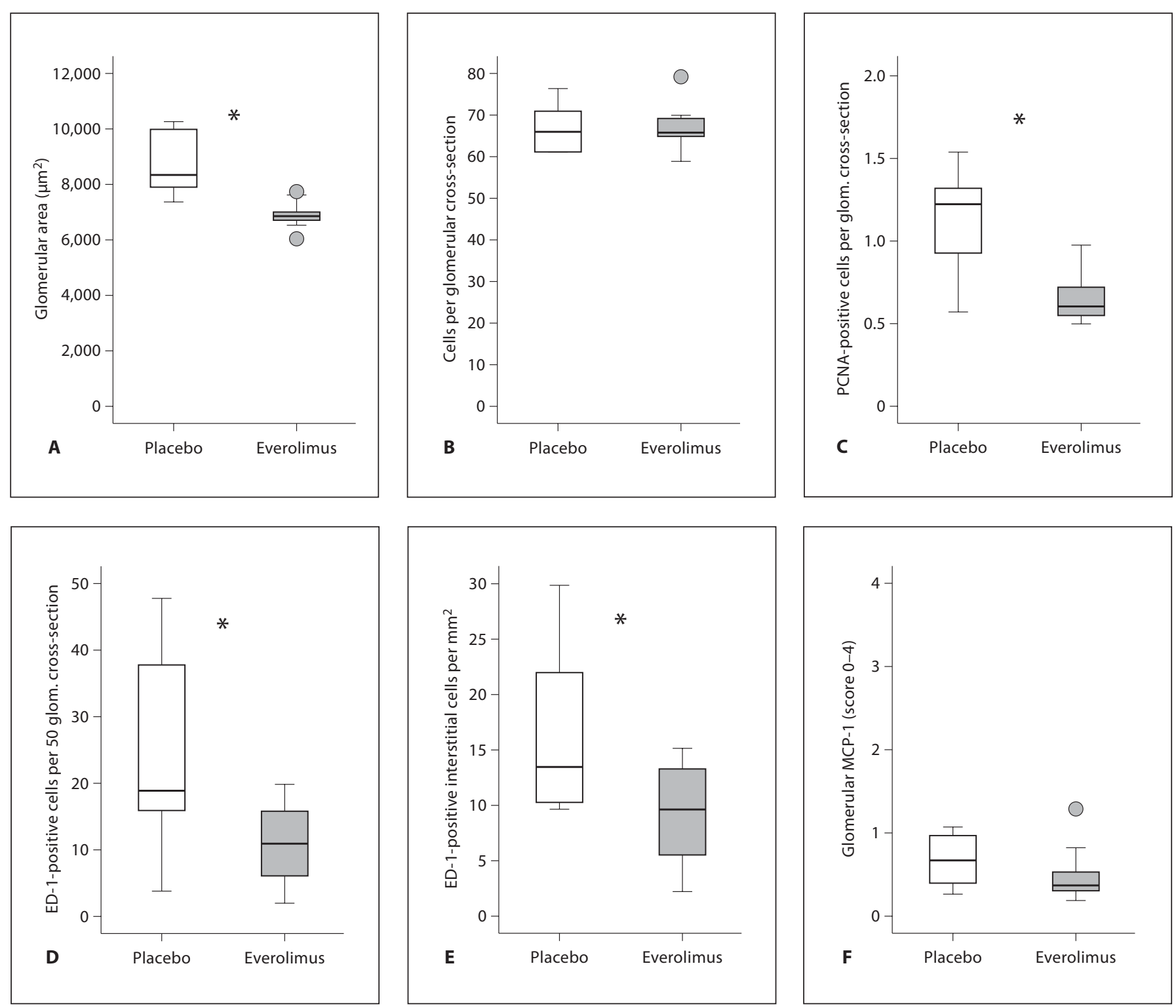

Fig. 6. Everolimus inhibits glomerular hypertrophy and influx of macrophages in chronic anti-Thy 1 nephritis. A Glomerular enlargement was reduced by everolimus treatment as assessed by measuring the area of glomerular cross-sections by computerized image quantification. ${ }^{*}$ Significant changes at $\mathrm{p}<0.05$. B In contrast, the cell number per glomerular cross-section was equal in both groups. C Glomerular proliferation as assessed by the number of PCNA-positive cells per glomerular cross-section was sig-

nificantly reduced by everolimus therapy. ${ }^{*}$ Significant changes at $\mathrm{p}<0.05$. D, E Influx of monocytes/macrophages as assessed by immunostaining for ED-1-positive cells was decreased by everolimus in both the glomerular (D) and the interstitial compartment (E). * Significant changes at $\mathrm{p}<0.05$. $\mathbf{F}$ The chemokine MCP-1 analyzed by immunostaining was evaluated by a score from 0 to 4 .

p70S6 kinase was significantly reduced by $26 \pm 5.5 \%$ in everolimus-treated rats compared to placebo-treated animals (fig. 5B).

To investigate the long-term impact of everolimus on the glomerular endothelium, we investigated glomerular

capillary rarefaction and the regulation of the important pro-angiogenic factor VEGF. Everolimus therapy significantly reduced glomerular VEGF immunostaining by $22 \pm 4 \%$ as indicated by semiquantitative scoring (fig. 5C). In addition, renal glomerular VEGF mRNA 

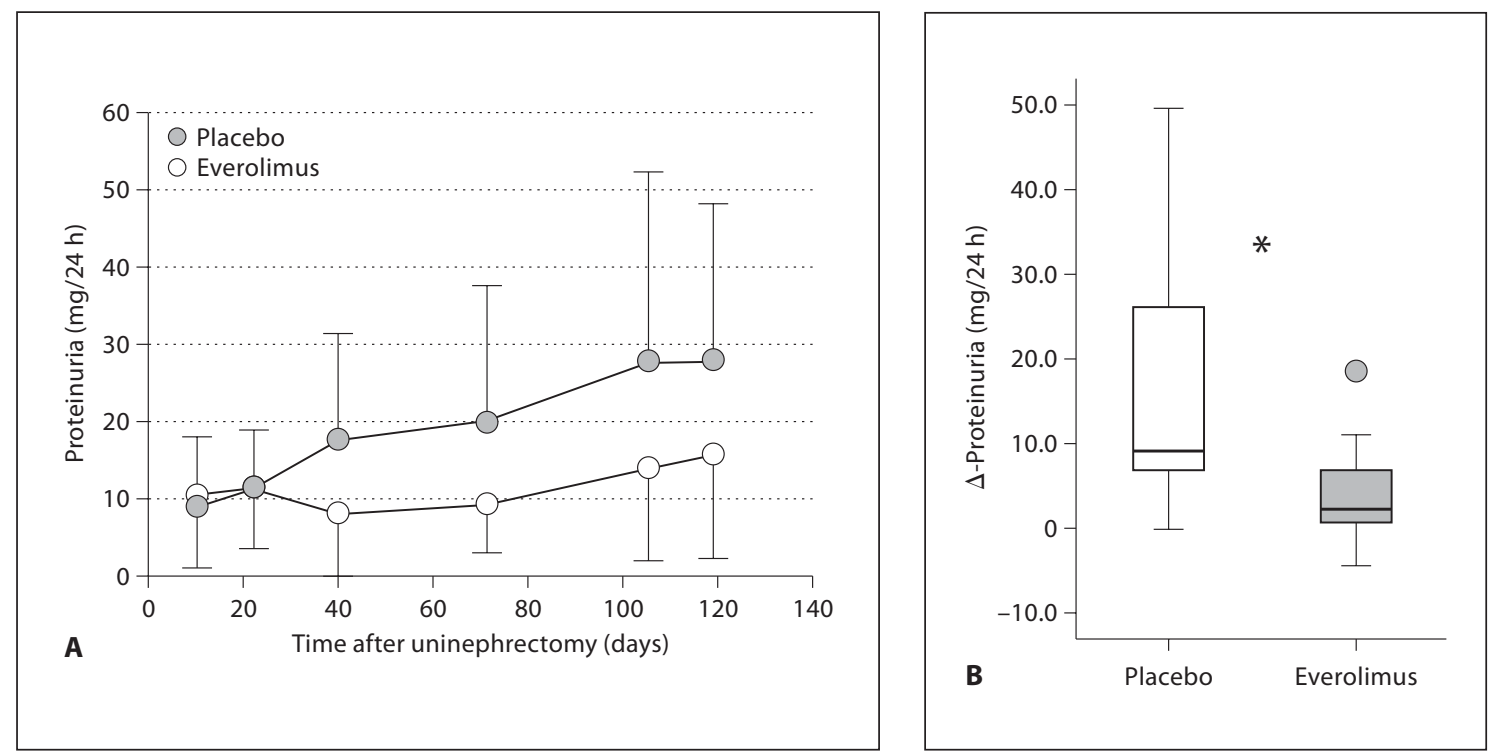

Fig. 7. Everolimus prevents proteinuria in chronic anti-Thyl nephritis. A In the time course of chronic anti-Thyl nephritis everolimus prevented an increase in proteinuria. B $\Delta$-proteinuria, calculated as the difference between the starting point and end of therapy, showed significantly less proteinuria in everolimus-treated animals. ${ }^{*}$ Significant changes at $\mathrm{p}<0.05$. C, D Everolimus therapy did not influence blood pressure as measured by the tailcuff method (C) or renal function as shown by comparable creatinine clearance (D).
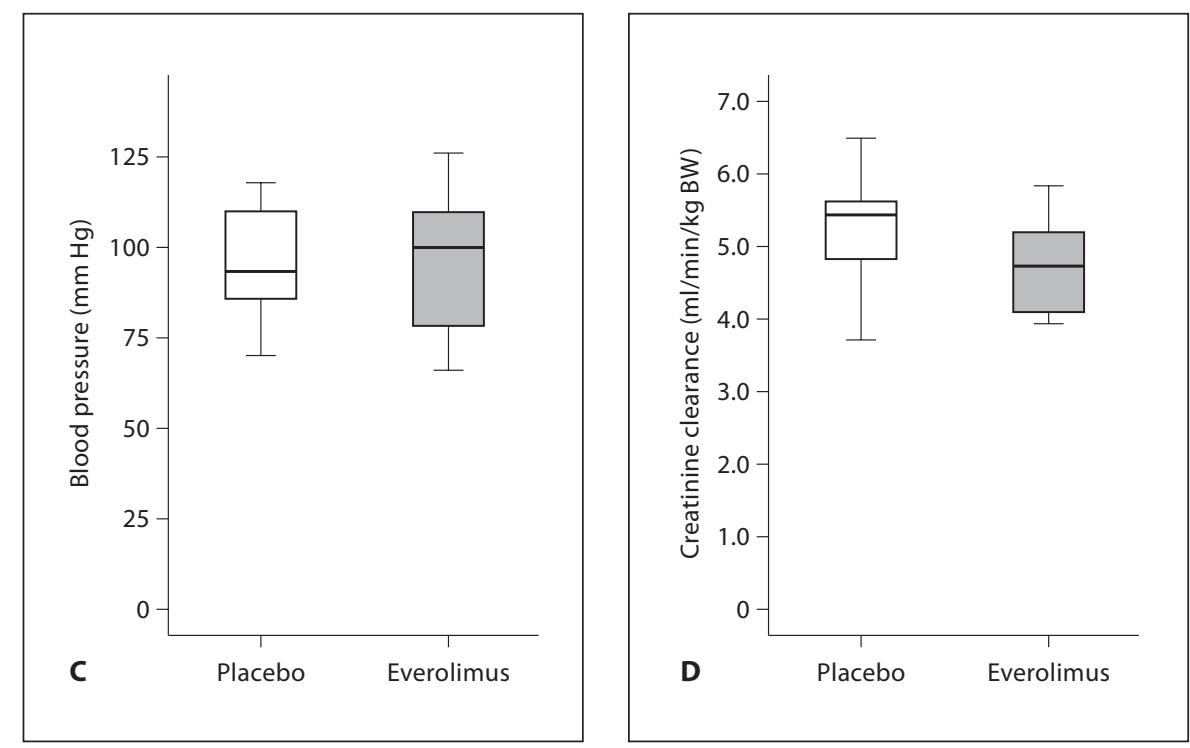

was significantly decreased by more than $50 \%$ in everolimus-treated animals compared to the vehicle-treated group as assessed via real-time PCR (fig. 5D). Despite these inhibitory effects on VEGF, glomerular capillary rarefaction as assessed by scoring of JG12 staining was not significantly affected by long-term everolimus treatment (fig. 5E).

\section{Everolimus Inhibits Hypertrophy and Inflammation in Chronic Anti-Thy1 Nephritis}

During chronic anti-Thyl nephritis, the glomerular area increased by $20 \pm 6 \%$ in vehicle-treated animals compared to age-matched healthy rats (about 7,000 $\mu \mathrm{m}^{2}$ as revealed by another of our studies, data not published; fig. 6A). Everolimus therapy prevented enlargement of the glomerular cross-sectional area (fig. 6A). Since glomerular cell number was not different in everolimus- and vehicle-treated rats, glomerular enlargement does not appear to be due to increased cell numbers (fig. 6B), but cellular hypertrophy does. Nevertheless, since everolimus is known for its antiproliferative effects, we also examined proliferative activity at the end of the study. In both groups only a low number of PCNA-positive cells was seen and 'low grade proliferation' was significantly re- 
duced in the everolimus-treated compared to vehicletreated rats (fig. 6C).

Inflammatory cells are important mediators of fibrosis induction, therefore influx of macrophages was investigated by immunohistochemistry. Influx of monocytes/ macrophages, as demonstrated by the number of ED1positive cells, was significantly decreased by more than $50 \%$ by everolimus treatment in both the glomerular and interstitial compartment (fig. 6D, E). Since the influx of monocytes/macrophages was decreased by mTOR inhibition, we also examined the chemokine MCP-1 by immunostaining, which was not different in the diseased glomeruli of everolimus- versus placebo-treated rats (fig. 6F).

\section{Everolimus Prevents Proteinuria in Chronic Anti-Thy1 Nephritis}

Proteinuria was analyzed as a marker of severity of nephropathy. At the beginning of treatment (on day 14), the animals were randomized to either the everolimus- or vehicle-treated group. Proteinuria increased with time after induction of chronic anti-Thyl nephritis in the vehicle-treated group (fig. 7A). This increase in proteinuria was prevented by everolimus therapy (fig. 7A). The $\Delta$ proteinuria showing the difference in proteinuria between the start and the end point of therapy was very small in the everolimus-treated group and significantly lower compared to vehicle-treated animals (fig. 7B). The beneficial effects of everolimus were not due to effects on blood pressure, since blood pressure was similar in both groups (fig. 7C). Renal function was not affected by everolimus therapy (fig. 7D).

\section{Discussion}

Unexpected adverse effects of mTOR inhibitors, such as the induction of proteinuria and renal deterioration [5, $29,30]$, have been described. In this context, we recently demonstrated the severe adverse effects of everolimus therapy in acute experimental mesangial proliferative glomerulonephritis in the rat (reversible anti-Thyl model) [8], where inhibition of capillary repair by everolimus in the especially vulnerable mesangiolytic phase and acute proliferative phase with the occurrence of microaneurysms seemed to be most critical. This acute reversible anti-Thyl model can be converted into a chronic mesangial proliferative glomerulonephritis model by uninephrectomy and the use of the anti-Thyl antibody 1-22-3 [12-14]. Hereby, the first mesangiolytic and hyper- proliferative phase is basically identical to the reversible anti-Thyl model, but instead of a complete healing reaction, glomerular hypertrophy, hypercellularity and matrix expansion slowly progress over the next few months. We hypothesized that treatment with everolimus starting after the acute proliferative phase with a largely intact glomerular capillary convolute could even be beneficial in the chronic anti-Thyl model.

Confirming our hypothesis, our major finding of this study is that everolimus therapy initiated 14 days after disease induction (at a time when glomerular endothelium and mesangium was restored) did not worsen the time course of experimental chronic mesangial proliferative glomerulonephritis, although the same drug doses were used as in the reversible anti-Thyl model [8] leading to clinically relevant drug levels $(6-10 \mathrm{ng} / \mathrm{ml})$. Rather, everolimus caused beneficial long-term effects after 4 months of therapy as indicated by inhibition of the formation of FSGS lesions, interstitial fibrosis, proteinuria, glomerular hypertrophy, and accumulation of macrophages/monocytes as well as matrix proteins as indicated by FN mRNA and protein. This inhibitory effect on glomerular FN production and accumulation seems to be independent of the TGF- $\beta$ system, since TGF- $\beta$ mRNA and its typical target gene PAI-1 mRNA were unchanged by everolimus. Whether an inhibition of matrix accumulation or the reduction of accumulating inflammatory cells, such as macrophages/monocytes, are a primary or a secondary result of mTOR inhibition in this disease course is speculative. Nevertheless, this anti-inflammatory effect is important to notice, since even the proinflammatory actions of mTOR inhibitors have been described in response to certain cytokines in in vitro experiments [Saemann MD, personal commun.]. Consistent with our results in this study, a recent study demonstrated a direct inhibitory effect of macrophage adhesion via rapamycin-induced inhibition of rho-associated kinase-1 [31] causing reduced macrophage chemotaxis and phagocytosis. As shown before, everolimus reduced the number of glomerular cells positive for phosphorylated p70S6 kinase and decreased the amount of glomerular VEGF mRNA and protein. This is a reproducible result as shown by mTOR inhibitor treatment in many models of renal disease $[8,32-34]$ as well as in human renal (transplant) patients, and might be very important either for side effects or the positive effects of mTOR inhibition, dependent on the pathophysiological situation.

VEGF is the major pro-angiogenic factor that plays an important role in the development, maintenance, and repair of the endothelium in the kidney, as shown by the 
elegant studies by Eremina et al. [35, 36] and others [37, 38]. To date, there is no other known cytokine which is equally tightly controlled within the glomerulus, and with which mice with glomerular selective deletion, haplo-insufficiency, isoform-specific deletion or overexpression of VEGF develop different but specific glomerular diseases [36]. Nevertheless, the consequences of VEGF alteration seem to depend on the specific requirements of the glomerulus. Since the everolimus-induced VEGF reduction in the chronic anti-Thyl model, starting after the 2nd week of disease induction, did not lead to any severe alterations in the capillary convolute and architecture, but in the acute phase of the anti-Thyl model with mesangiolytic, microaneurysmatic glomeruli, the same therapy was detrimental. It even cannot be excluded that VEGF reduction may be a causal part of this beneficial influence of everolimus on the time course of our disease model, when considering the adverse renal outcome in transgenic mice overexpressing VEGF in podocytes [36] or in transgenic rabbits overexpressing the human VEGF isoform in the kidney and liver [39].

The beneficial effects of everolimus are consistent with the results in other published models of glomerular diseases, such as the aminonucleoside nephrosis model [9], the mercuric chloride-induced glomerulopathy [40] or in experimental membranous nephropathy [41]. Where the glomerular architecture stays intact, no severe combined mesangial and endothelial (glomerular) lesion needs to be repaired. In contrast, everolimus therapy also worsened the time course of disease in a chronic model of low nephron number, the 5/6 nephrectomy model, in which both glomerular endothelial and mesangial injury were demonstrated [Vogelbacher R, et al., Transplantation, in press].

In conclusion, our results in a chronic mesangial proliferative glomerulonephritis model in the rat demonstrate that mTOR inhibition at clinically relevant drug levels (6-9 $\mathrm{ng} / \mathrm{ml})$ can be beneficial for the time course of disease by impairing proteinuria, the occurrence of FSGS lesions, interstitial fibrosis, glomerular hypertrophy, matrix accumulation and glomerular inflammation. This study further confirms our previous observation that the adverse effects of mTOR inhibition appear to be linked to severe combined glomerular lesions needing a timely repair of the capillary convolute.

\section{Acknowledgments}

This work was supported by Novartis Pharma Basel, by the Deutsche Forschungsgemeinschaft (SFB 423 - Nierenschäden; TP B6 and TP B8) and by the Interdisciplinary Center for Clinical Research at the University Hospital of the University of ErlangenNuremberg (TP A12). The authors are indebted to Susanne Weber, Andrea Braun, Tanja Christ, Birgit Hausknecht and Andrea Lüdke for their technical help.

\section{References}

1 Schuler W, Sedrani R, Cottens S, Haberlin B, Schulz M, Schuurman HJ, Zenke G, Zerwes HG, Schreier MH: SDZ RAD, a new rapamycin derivative: pharmacological properties in vitro and in vivo. Transplantation 1997; 64:36-42.

2 Nashan B: Review of the proliferation inhibitor everolimus. Expert Opin Investig Drugs 2002;11:1845-1857.

3 Sehgal SN, Camardo JS, Scarola JA, Maida BT: Rapamycin (sirolimus, rapamune). Curr Opin Nephrol Hypertens 1995;4:482-487.

4 Dittrich E, Schmaldienst S, Soleiman A, Horl WH, Pohanka E: Rapamycin-associated post-transplantation glomerulonephritis and its remission after reintroduction of calcineurin-inhibitor therapy. Transpl Int 2004; 17:215-220.

5 Butani L: Investigation of pediatric renal transplant recipients with heavy proteinuria after sirolimus rescue. Transplantation 2004;78:1362-1366.
6 Diekmann F, Budde K, Oppenheimer F, Fritsche L, Neumayer HH, Campistol JM: Predictors of success in conversion from calcineurin inhibitor to sirolimus in chronic allograft dysfunction. Am J Transplant 2004; 4:1869-1875.

7 Fervenza FC, Fitzpatrick PM, Mertz J, Erickson SB, Liggett S, Popham S, Wochos DN, Synhavsky A, Hippler S, Larson TS, Bagniewski SM, Velosa JA: Acute rapamycin nephrotoxicity in native kidneys of patients with chronic glomerulopathies. Nephrol Dial Transplant 2004;19:1288-1292.

8 Daniel C, Renders L, Amann K, Schulze-Lohoff E, Hauser IA, Hugo C: Mechanisms of everolimus-induced glomerulosclerosis after glomerular injury in the rat. Am J Transplant 2005;5:2849-2861.

9 Daniel C, Ziswiler R, Frey B, Pfister M, Marti HP: Proinflammatory effects in experimental mesangial proliferative glomerulonephritis of the immunosuppressive agent SDZ RAD, a rapamycin derivative. Exp Nephrol 2000;8:52-62.
10 Mohacsi PJ, Tuller D, Hulliger B, Wijngaard PL: Different inhibitory effects of immunosuppressive drugs on human and rat aortic smooth muscle and endothelial cell proliferation stimulated by platelet-derived growth factor or endothelial cell growth factor. J Heart Lung Transplant 1997;16:484492.

11 Wang W, Chan YH, Lee W, Chan L: Effect of rapamycin and FK506 on mesangial cell proliferation. Transplant Proc 2001;33:10361037.

12 Kawachi H, Oite T, Shimizu F: Quantitative study of mesangial injury with proteinuria induced by monoclonal antibody 1-22-3. Clin Exp Immunol 1993;92:342-346.

13 Shimizu F, Kawachi H, Orikasa M: Role of mesangial cell damage in progressive renal disease. Kidney Blood Press Res 1999;22: 5-12. 
14 Kawachi H, Orikasa M, Matsui K, Iwanaga T, Toyabe S, Oite T, Shimizu F: Epitope-specific induction of mesangial lesions with proteinuria by a MoAb against mesangial cell surface antigen. Clin Exp Immunol 1992;88:399-404.

15 Wu M, Wahl PR, Le Hir M, Wackerle-Men Y, Wuthrich RP, Serra AL: Everolimus retards cyst growth and preserves kidney function in a rodent model for polycystic kidney disease. Kidney Blood Press Res 2007;30:253259.

16 Nangaku M, Alpers CE, Pippin J, Shankland SJ, Adler S, Kurokawa K, Couser WG, Johnson RJ: A new model of renal microvascular endothelial injury. Kidney Int 1997;52:182194.

17 Iruela-Arispe L, Gordon K, Hugo C, Duijvestijn AM, Claffey KP, Reilly $M$, Couser WG, Alpers CE, Johnson RJ: Participation of glomerular endothelial cells in the capillary repair of glomerulonephritis. Am J Pathol 1995; 147:1715-1727.

18 Hugo C, Hugo C, Pichler R, Gordon K, Schmidt R, Amieva M, Couser WG, Furthmayr H, Johnson RJ: The cytoskeletal linking proteins, moesin and radixin, are upregulated by platelet-derived growth factor, but not basic fibroblast growth factor in experimental mesangial proliferative glomerulonephritis. J Clin Invest 1996;97:2499-2508.

19 Matsui K, Nagy-Bojarsky K, Laakkonen P, Krieger S, Mechtler K, Uchida S, Geleff S, Kang DH, Johnson RJ, Kerjaschki D: Lymphatic microvessels in the rat remnant kidney model of renal fibrosis: aminopeptidase $\mathrm{p}$ and podoplanin are discriminatory markers for endothelial cells of blood and lymphatic vessels. J Am Soc Nephrol 2003;14: 1981-1989.

20 Kang DH, Joly AH, Oh SW, Hugo C, Kerjaschki D, Gordon KL, Mazzali M, Jefferson JA, Hughes J, Madsen KM, Schreiner GF, Johnson RJ: Impaired angiogenesis in the remnant kidney model: I. Potential role of vascular endothelial growth factor and thrombospondin-1. J Am Soc Nephrol 2001; 12:1434-1447.

21 Daniel C, Pippin J, Shankland SJ, Hugo C: The rapamycin derivative RAD inhibits mesangial cell migration through the CDK-inhibitor p27KIP1. Lab Invest 2004;84:588596.

22 Hugo C, Shankland SJ, Pichler RH, Couser WG, Johnson RJ: Thrombospondin 1 precedes and predicts the development of tubulointerstitial fibrosis in glomerular disease in the rat. Kidney Int 1998;53:302-311.
23 Johnson RJ: The glomerular response to injury: progression or resolution? Kidney Int 1994;45:1769-1782.

24 Riley SG, Steadman R, Williams JD, Floege J, Phillips AO: Augmentation of kidney injury by basic fibroblast growth factor or platelet-derived growth factor does not induce progressive diabetic nephropathy in the Goto Kakizaki model of non-insulin-dependent diabetes. J Lab Clin Med 1999;134:304312.

25 Daniel C, Takabatake Y, Mizui M, Isaka Y, Kawashi H, Rupprecht H, Imai E, Hugo C: Antisense oligonucleotides against thrombospondin-1 inhibit activation of tgf-beta in fibrotic renal disease in the rat in vivo. Am J Pathol 2003;163:1185-1192.

26 Yan M, Schneider J, Gear R, Lu F, LaDow K, Warshawsky D, Heffelfinger SC: Expression of angiogenic factors is upregulated in DMBA-induced rat mammary pathologies. Pathobiology 2004;71:253-260.

27 Ruiz V, Ordonez RM, Berumen J, Ramirez R, Uhal B, Becerril C, Pardo A, Selman M: Unbalanced collagenases/TIMP-1 expression and epithelial apoptosis in experimental lung fibrosis. Am J Physiol Lung Cell Mol Physiol 2003;285:L1026-L1036.

28 Jacobi J, Porst M, Cordasic N, Namer B, Schmieder RE, Eckardt KU, Hilgers KF: Subtotal nephrectomy impairs ischemia-induced angiogenesis and hindlimb re-perfusion in rats. Kidney Int 2006;69:2013-2021.

29 Rangan GK: Sirolimus-associated proteinuria and renal dysfunction. Drug Saf 2006; 29:1153-1161.

30 Ruiz JC, Campistol JM, Sanchez-Fructuoso A, Rivera C, Oliver J, Ramos D, Campos B, Arias M, Diekmann F: Increase of proteinuria after conversion from calcineurin inhibitor to sirolimus-based treatment in kidney transplant patients with chronic allograft dysfunction. Nephrol Dial Transplant 2006;21:3252-3257.

31 Fox R, Nhan TQ, Law GL, Morris DR, Liles WC, Schwartz SM: PSGL-1 and mTOR regulate translation of ROCK-1 and physiological functions of macrophages. EMBO J 2007;26: 505-515.
32 Guba M, von Breitenbuch P, Steinbauer M, Koehl G, Flegel S, Hornung M, Bruns CJ, Zuelke C, Farkas S, Anthuber M, Jauch KW, Geissler EK: Rapamycin inhibits primary and metastatic tumor growth by antiangiogenesis: involvement of vascular endothelial growth factor. Nat Med 2002;8:128-135.

33 Luan FL, Ding R, Sharma VK, Chon WJ, Lagman M, Suthanthiran M: Rapamycin is an effective inhibitor of human renal cancer metastasis. Kidney Int 2003;63:917-926.

34 Lieberthal W, Fuhro R, Andry CC, Rennke H, Abernathy VE, Koh JS, Valeri R, Levine JS: Rapamycin impairs recovery from acute renal failure: role of cell-cycle arrest and apoptosis of tubular cells. Am J Physiol Renal Physiol 2001;281:F693-F706.

35 Eremina V, Quaggin SE: The role of VEGF-A in glomerular development and function. Curr Opin Nephrol Hypertens 2004;13:915.

36 Eremina V, Sood M, Haigh J, Nagy A, Lajoie G, Ferrara N, Gerber HP, Kikkawa Y, Miner JH, Quaggin SE: Glomerular-specific alterations of VEGF-A expression lead to distinct congenital and acquired renal diseases. J Clin Invest 2003;111:707-716.

37 Ostendorf T, Kunter U, Eitner F, Loos A, Regele $\mathrm{H}$, Kerjaschki D, Henninger DD, Janjic $\mathrm{N}$, Floege J: VEGF(165) mediates glomerular endothelial repair. J Clin Invest 1999;104: 913-923.

38 Masuda Y, Shimizu A, Mori T, Ishiwata T, Kitamura H, Ohashi R, Ishizaki M, Asano G, Sugisaki Y, Yamanaka N: Vascular endothelial growth factor enhances glomerular capillary repair and accelerates resolution of experimentally induced glomerulonephritis. Am J Pathol 2001;159:599-608.

39 Liu E, Morimoto M, Kitajima S, Koike T, Yu Y, Shiiki H, Nagata M, Watanabe T, Fan J: Increased expression of vascular endothelial growth factor in kidney leads to progressive impairment of glomerular functions. J Am Soc Nephrol 2007; 18:2094-2104.

40 Hermann P, Poschmann G, Bolliger S, Meyer D, Schuurman HJ: Mercuric chloride-induced glomerulopathy in BN-rats: application to preclinical drug testing. Transplant Proc 1993;25:2826-2827.

41 Bonegio RG, Fuhro R, Wang Z, Valeri CR Andry C, Salant DJ, Lieberthal W: Rapamycin ameliorates proteinuria-associated tubulointerstitial inflammation and fibrosis in experimental membranous nephropathy. J Am Soc Nephrol 2005;16:2063-2072. 